\title{
The Elastic Ligation of Internal Hemorrhoids: Where Are We Now?
}

\author{
H. Abid ${ }^{1,2}$, G. Ousseur ${ }^{1,2}$, R. Benjira1,2, M. Lahlali1,2, A. Lamine ${ }^{1,2}$, N. Lahmidani1 ${ }^{1,2}$, M. El Yousfi ${ }^{1,2}$, \\ N. Aqodad1,2, D. Benajah',2, A. Ibrahimi1,2, M. El Abkari'1,2
}

${ }^{1}$ Hepato-Gastroenterology Department, Hassan II University Medical Center, Fez, Morocco

${ }^{2}$ Faculty of Medicine, Sidi Mohammed Ben Abdellah University, Fez, Morocco

Email: abidhakima@hotmail.com

How to cite this paper: Abid, H., Ousseur, G., Benjira, R., Lahlali, M., Lamine, A., Lahmidani, N., El Yousfi, M., Aqodad, N., Benajah, D., Ibrahimi, A. and El Abkari, M. (2019) The Elastic Ligation of Internal Hemorrhoids: Where Are We Now? Open Journal of Gastroenterology, 9, 28-35. https://doi.org/10.4236/ojgas.2019.91005

Received: December 14, 2018

Accepted: January 21, 2019

Published: January 24, 2019

Copyright $\odot 2019$ by author(s) and Scientific Research Publishing Inc. This work is licensed under the Creative Commons Attribution International License (CC BY 4.0).

http://creativecommons.org/licenses/by/4.0/

\begin{abstract}
Hemorrhoidal disease requires different means of treatment: medical, surgical and instrumental. Among these, the elastic ligature seems to be an effective and widespread technique in the world. The aim of our study is to report our experience in methodology and short and medium term results of ligation in a population that strongly fears surgery. Patients and Methods: It's about a retrospective and descriptive study of 208 outpatients treated with elastic ligations for symptomatic internal hemorrhoids. These patients were collected over a period of 15 years (October 2003-April 2018) at the gastroenterology department of Hassan II CHU in Fez. Results: The average age of our patients was 50.3 years [20 - 82 years] with a sex-ratio M/F of 2.85 . The clinical signs were dominated by rectorrhagia (99\%) complicated by anemia in 116 cases requiring blood transfusion in 72 cases, followed by proctalgia (27.4\%). Transit disorders were noted in 65 patients (31.2\%). Indications for elastic ligation were symptomatic internal hemorrhoids Grade 3 (65.4\%) and Grade $2(34.6 \%)$. The average number of ligation sessions that achieved the therapeutic goal was 2.30 sessions [ 1 - 4]. The average number of rings per session was 3.1 rings [ $1-6]$. Moderate to severe pain was reported by 20 patients (9.6\%) mostly within 6 hours of ligation. Minimal rectorrhagia was reported in 33 cases $(15.8 \%)$. There were no major complications who required a hospitalization. The success rate was $80.7 \%(n=168)$. Surgery was performed in 31 patients (14.9\%) and sclerosis in 7 patients (3.3\%). Conclusion: The elastic ligation of internal hemorrhoids remains an effective and inexpensive technique when it comes to the treatment of symptomatic internal hemorrhoids of Grade 2 to 3 . The results obtained in our study were very reassuring and motivating.
\end{abstract}

\section{Keywords}

Internal Hemorrhoids, Elastic Ligature, Evolution 


\section{Introduction}

Hemorrhoidal pathology is one of the most common causes of gastroenterology consultation. It is a benign condition that is expressed by intermittent functional manifestations. Internal hemorrhoids manifest themselves by rectorrhagia and/or prolapse, more rarely by thrombosis. The treatment of internal hemorrhoids involves many medical, instrumental and surgical methods, the choice of which must be adapted to each patient according to the stage and symptoms expressed. Instrumental treatment, of which there are several methods, currently occupies an important place in the management of hemorrhoidal disease. The aim of the instrumental treatment is not to restore a normal anatomy, but simply to permanently omit the symptoms by creating a cicatricial fibrosis which fixes the mucosa in the deep plane and reduces the vascularization, thus allowing the symptomatic relief (rectorragies) and the correction of a possible anatomical defect (prolapse). Among the instrumental treatments, elastic ligation is considered an efficient technique widely used in the world.

\section{Aim of the Study}

Our objective is to present a moroccan series of patients with symptomatic internal hemorrhoids treated by elastic ligation in the hepatogastroenterology department of Hassan II university hospital in Fez. Through this series, we seek to get a general idea of the epidemiological characteristics of internal hemorrhoids, and to report the service experience regarding elastic ligation, while emphasizing short-term and long-term efficiency as well as complications of the technique and compare our results with those present in national and international literature.

\section{Materials and Methods}

This is a retrospective and descriptive study involving 208 patients treated by elastic ligatures for symptomatic internal hemorrhoids, Grade 2 or 3. These patients were collected over a period of 15 years [October 2003-April 2018] at Hassan II University Hospital in Fez, Morocco. Dietary and defecatory hygiene measures were prescribed to all patients. A transit regulator has been prescribed for patients suffering from constipation. Elastic ligation was performed in patients who did not improve after well-conducted medical treatment, or with anemia related to hemorrhoidal disease. Coagulation tests were performed in all patients before the procedure.

All our patients were informed about the indication of the ligature, the progress of the technique, the incidents and also the potential complications. All our patients received post-ligation antibiotic therapy (metronidazole $1.5 \mathrm{~g}$ daily) combined with analgesics for $72 \mathrm{~h}$.

The therapeutic goal was defined by the disappearance of clinical symptoms after 1 to 4 sessions of ligations spaced 3 to 4 weeks. 
The variables were retrospectively collected in a computer database then their statistical analysis was performed using SPSS 20.0.

There were no limitations of this study.

\subsection{Definitions}

Short term success: the disappearance of the symptoms after one to four ligation sessions.

Long term succes: non reappearance of symptoms more than one year after the end of ligation protocol.

Failure: the lack of clinical response after a maximum of 4 ligation sessions.

Recurrence rate: the reappearance of symptoms after the end of the ligation protocol.Evidently, it has been clarified exclusively amongst patients who have responded to the ligation protocol successfully.

\subsection{Technique}

The purpose of the ligature elastic is to achieve a "sclerosis nail" fixing mucous membrane with free fibers. The method consists of set up an elastic ring in the insensitive cylindrical area suprapectine. The suction apparatus is preferred to the claw device as it does not cause mucosal breakthrough and allows for better control at view. The apparatus comprises two cylinders whose outer is mobile. An elastic band (or vinyl ring in case of allergy latex) is placed using an introducer cone on the inner cylinder. The device is connected to a suction. The mucosa is sucked into the inner cylinder. The outer cylinder is mobilized forward sliding on the inner and pushes the elastic at the base of the pedicle aspirated. The ligated tissues become necrotic by forming a eschar which heals in three weeks (Figure 1 and Figure 2) [1].

\section{Results}

The average age of our patients was 50.3 years [20 - 82 years] with a large male predominance $($ sex ratio $=2.85)$. A personal history of portal hypertension was found in two patients, one hemorrhoidectomy in one patient, an ulcerative colitis in two patients, one patient underwent surgery for a sigmoid adenocarcinoma, and one patient underwent an anal fistula surgery. Clinical

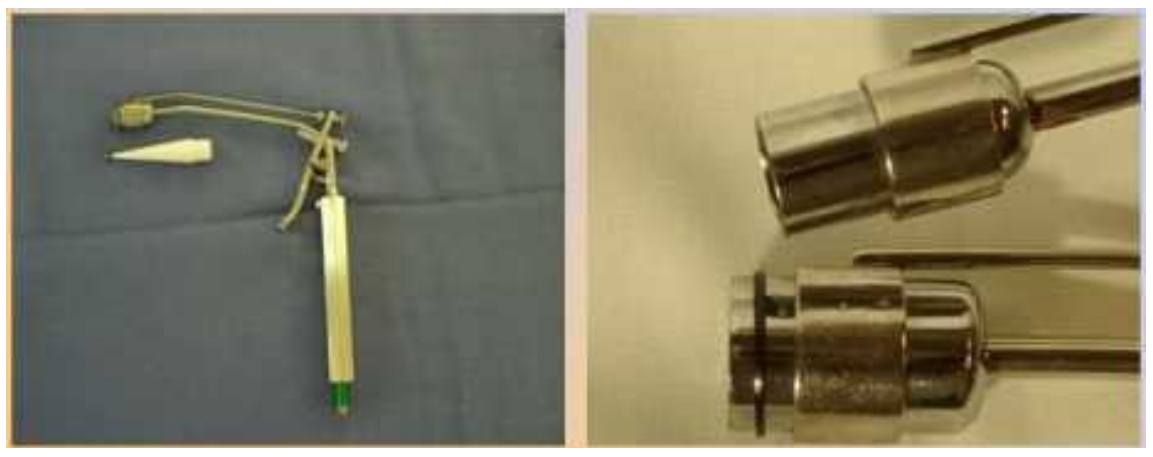

Figure 1. Material of the elastic ligation of internal hemorrhoids. 

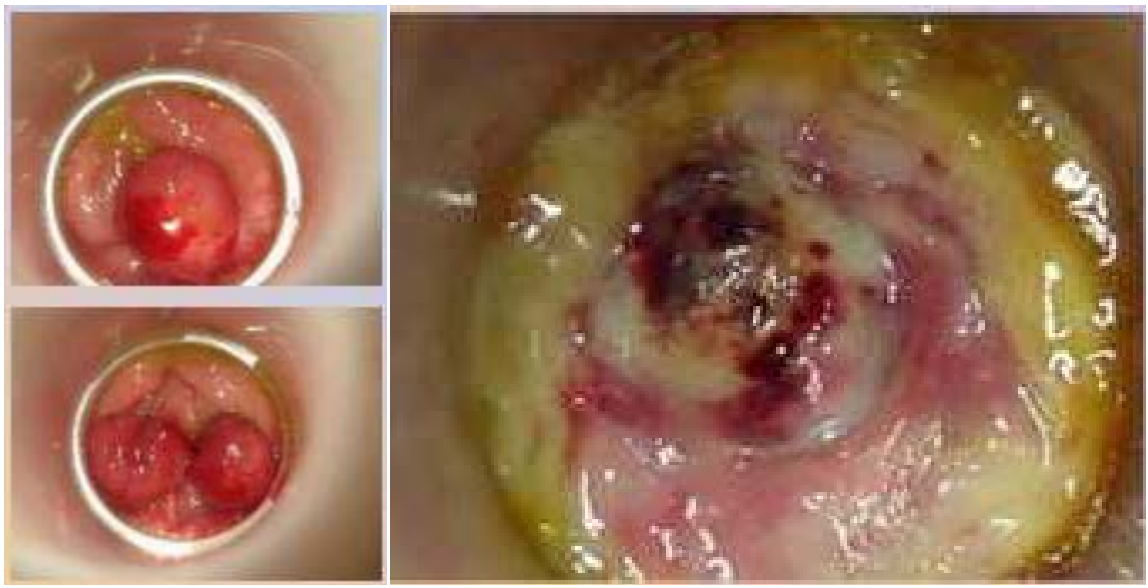

Figure 2. Immediate appearance post-ligature/appearance after fall of eschar.

symptomatology was dominated by rectorragies ( $\mathrm{n}=206$ or $99 \%$ of cases) and prolapse ( $\mathrm{n}=176,84.6 \%$ of cases). Anemia was noted in $55.7 \%$ of cases $(\mathrm{n}=$ $116)$, with recourse to transfusion in $35.1 \%$ of patients $(n=72)$. The proctological examination found internal hemorrhoids Grade II in $34.6 \%(\mathrm{n}=$ 72) and Grade III in 65.4\% $(\mathrm{n}=136)$. Endoscopic exploration by total colonoscopy was performed in all patients aged over 45 years, and in patients with profound anemia to eliminate another underlying cause. Endoscopic exploration revealed the presence of polyps in 3 patients, two of whom had high grade dysplasia in the histological analysis. The assessment of blood tests based on prothrombin rate and platelet count, was considered good in all patients (Table 1). The average number of sessions that achieved the therapeutic goal was 2.3 sessions [ $1-4$ sessions]. The average number of rings per session was 3.1 [1 - 6 rings]. Minor complications were notified in 42 patients (20.2\%) with minimal bleeding in $15.3 \%$ of patients $(\mathrm{n}=32$ ), and moderate pain in $9.6 \%$ of patients $(n=20)$ who responded to ambulatory symptomatic treatment, without requiring hospitalization.

The success rate was $80.7 \%(\mathrm{n}=168)$. After an average follow-up of 42 months [ 4 months - 6 years], the recurrence rate was $25 \%(n=52)$, requiring the use of instrumental treatment in 15 patients (7.2\% of cases), with surgical aid in 31 patients (15\% of cases), while the remaining 6 patients were medically treated successfully (Figure 3 ).

\section{Discussion}

The instrumental treatment of hemorrhoids includes methods applying a chemical or a physical process that can treat internal hemorrhoidal disease responsible for rectorragies or Grade 2 or 3 prolapse [1]. The principle common to these instrumental treatments consists of causing at the top of the internal hemorrhoidal plexuses a cicatricial fibrosis of which the consequences are two fold: fixation of the anal mucosa on the underlying muscular plane and closure of the submucosal vascular network coming from the superior rectal artery. In 
Table 1. Clinical characteristics of the enrolled patients.

\begin{tabular}{cc}
\hline & Patients $(\mathbf{n}=\mathbf{1 4 8})$ \\
\hline Mean age & 50.3 years $[20-82$ years $]$ \\
Sex & \\
Male & $154(74 \%)$ \\
Female & $54(26 \%)$ \\
Symptoms & \\
Rectorrhagies & $206(99 \%)$ \\
Prolapse & $176(84.6 \%)$ \\
Constipation & $61(29.3 \%)$ \\
Proctalgia & $26(12.5 \%)$ \\
internal hemorrhoids grade & \\
Grade II & $72(34.6 \%)$ \\
Grade III & $136(65.4 \%)$ \\
\hline
\end{tabular}

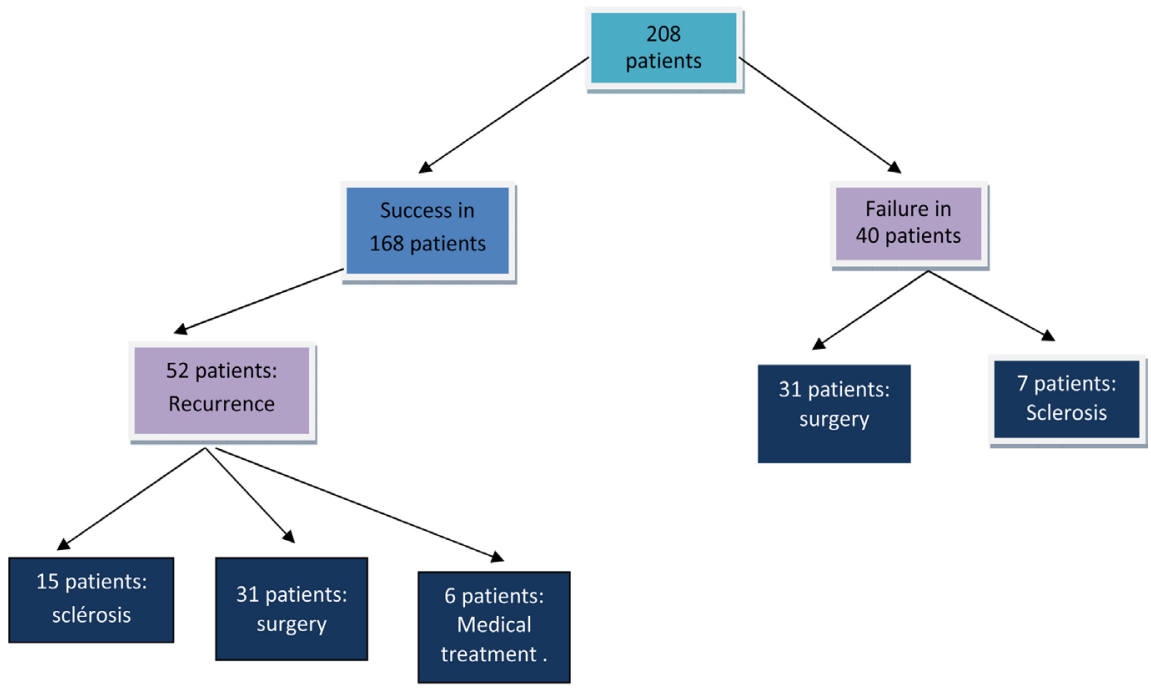

Figure 3. Flowchart of the enrolled patients.

addition, elastic ligation achieves a partial reduction of the internal hemorrhoidal volume, with a more pronounced effect on prolapse [2].

The elastic ligation is introduced in France by Soullard in 1966, it requires a ligator and a suction system. It must be made at least one centimeter above the pectinate line. The ligated tissues lead to the formation of an eschar of which the cicatrization is done in 2 to 3 weeks. The rhythm between two successive ligatures is one session every 4 weeks, limiting itself to a total of 3 or 4 sessions. The results after a triple ligation in one session [3] are comparable to those of three ligatures in three successive sessions (Grade A) [4].

In our series, the average number of sessions that reached the therapeutic goal was 2.3 sessions ( 1 - 4 sessions) while the average number of rings per session was 3.1 ( 1 - 6 rings). In a series of IbnSina university hospital of Rabat covering 191 patients, $4.76 \%$ of whom were treated by elastic ligature, the average number of sessions was 2.6 [5]. 
Elastic ligations are recommended in case of bleeding (Grade A) [5] and moderate haemorrhoidal prolapse, or limited to a single bundle in cases of internal hemorrhoids Grade 2 and 3 (Grade B) [6] [7]. Almost all of our patients (99\%) had rectorragies, with prolapse in $84.6 \%$ of patients. In the Rabat series, rectorragies were noted in $96.85 \%$ of cases, proctalgia in $54 \%$ of cases and an anemic syndrome was noted in $11.5 \%$ of patients [5].

In case of rectorrhagies without prolapse, elastic ligation should only be proposed as second-line after failure of a treatment normalizing intestinal transit which has a similar efficiency at 3 months [6] [7] [8] and probably after failure of infrared photocoagulation or sclerotherapy, with comparable results with fewer side effects and complications [7] [8] [9]. When the dominant symptom is prolapse, an immediate ligature can be proposed because of its demonstrated superiority, especially in the long term [9] [10]. The superiority of ligatures is clearer in case of prolapse, in fewer sessions than for other techniques [8] [9] [10]. In the absence of contraindication, elastic ligations may be proposed before surgery but they are less effective on circular Grade 3 prolapse and ineffective on Grade 4 prolapse [11] [12] [13].

In the short term, symptoms disappear or improve in $70 \%$ to $90 \%$ of cases [14] [15]. At one year and especially three years, the efficiency of elastic ligations is significantly greater than that of infrared photocoagulation, $40 \%$ at one year [14] [16] [17] and sclerosing injections, $28 \%$ at 2 years [11].

In our series, the success rate was $80.7 \%(n=168)$ which is consistent with the literature data. In the series of J. C. Bernal and Al the success rate is at $74 \%$ while it is at $99.7 \%$ in William W. H. Rudd's series [18]. In the long term (average follow-up of 42 months [ 4 months- 6 years]), the recurrence rate in our patients was $25 \%(\mathrm{n}=52)$, it occurred mainly in patients with internal Grade 3 hemorrhoids initially.

In the Rabat series, the long-term evolution was good in $62 \%$ of cases, with a less good evolution and appearance of external hemorrhoidal pathologies and fissures requiring only medical treatment in $19 \%$ of cases. Recurrence was noted in $19 \%$ of cases within an average of 13 months, this recurrence was controlled by another instrumental treatment technique in $65 \%$ of cases and surgery in $35 \%$ of cases (immediate in 9 patients and after failure of the 2nd attempt of the instrumental treatment at 2 patients) [5].

Complications are possible ( $1 \%$ to $5 \%$ of cases) with internal or external haemorrhoidal thrombosis, dysuria or, more rarely, acute urinary retention, major pain, or perianal abscess [19] [20]. Severe haemorrhaging up to 15 days after ligation requiring haemostasis is reported in $0.2 \%$ to $1.2 \%$ of cases [19]. More than 20 cases of very serious infectious complications, mainly pelvic cellulitis, have been published in the literature [20]. The patient should be warned of early signs of anorectal pain and urinary disorders between the 2nd and 7th days after ligation. Prophylactic antibiotic treatment with metronidazole is recommended as well as the prescription of analgesics Class 1 or 2 [2]. 
In our series, we found minimal rectorrhagies in $15.3 \%$ of patients $(n=33)$, and moderate pain in $9.6 \%$ of patients $(n=20)$; despite the frequency of these complications, they were generally benign, having responded to ambulatory symptomatic treatment.

In the Rabat University Hospital series, immediate complications were represented by pain and bleeding in $31.4 \%$ of cases [5].

\section{Conclusions}

The elastic ligation is a safe, economical and effective technique for the treatment of symptomatic internal hemorrhoids especially Grade 3, allowing good results in the short and long term. It allows a high rate of success compared to other instrumental techniques with a recurrence rate and low risk of complications.

In our series, ligation resulted in an initial success of $80.7 \%$, with relatively low rates of minor complications $(20.2 \%)$ and an acceptable recurrence rate (25\%).

\section{Conflicts of Interest}

The authors declare no conflicts of interest regarding the publication of this paper.

\section{References}

[1] Coulom, P. (2009) Non Surgical Therapy of Hemorrhoids. Gastroentérologie Clinique et Biologique, 33, F20-F26.

[2] Abramowitz, L., et al. (2001) Recommandations pour la pratique clinique sur le traitement de la maladie hémorroïdaire. Gastroentérologie Clinique et Biologique, 25, 674-702.

[3] Poon, G.P., Chu, K.W., Lau, W.Y., Lee, J.M., Yeung, C., Fan, S.T., Yiu, T.F., Wong, S.H. and Wong, K.K. (1986) Conventional versus Triple Rubber Band Ligation for Hemorrhoids. A Prospective, Randomized Trial. Diseases of the Colon \& Rectum, 29, 836-838.

[4] Higuero, T., Abramowitz, L., Staumont, G., et al. (2014) Clinical Practice Guidelines for the Treatment of Hemorrhoid Disease. Société Nationale Françoise de Colo-Proctologie (SNFCP), in Press.

[5] Lagdali, N., Ajana, F.Z., Benelbardadi, I., Afifi, R. and Essaid, A. (2016) Traitement instrumental des hémorroïdes. RMMAD No. 23 décembre.

[6] Jiang, Z., Tang, W., Cao, J., et al. (2001) Double-Blind Randomized Clinical Trial in Evaluating the Efficacy and Safety of Rectum Mucosa Protector in the Treatment of Acute Hemorrhoids. Chinese Journal of Surgery, 39, 53-55.

[7] Ho, Y.H., Tan, M. and Seow-Choen, F. (2000) Micronized Purified Flavonidic Fraction Compared Favorably with Rubber Band Ligation and Fiberalone in the Management of Bleeding Hemorrhoids: Randomized Controlled Trial. Diseases of the Colon \& Rectum, 43, 66-69. https://doi.org/10.1007/BF02237246

[8] Johanson, J.F. and Rimm, A. (1992) Optimal Nonsurgical Treatment of Hemorrhoids: A Comparative Analysis of Infrared Coagulation, Rubber Band Ligation, and Injection Sclerotherapy. The American Journal of Gastroenterology, 87, 1600-1606.

[9] Gupta, P.J. (2003) Infrared Coagulation versus Rubber Band Ligation in Early Stage 
Hemorrhoids. Brazilian Journal of Medical and Biological Research, 36, 1433-1439. https://doi.org/10.1590/S0100-879X2003001000022

[10] MacRae, H.M. and McLeod, R.S. (1995) Comparison of Hemorrhoidal Treatment Modalities. A Metaanalysis. Diseases of the Colon \& Rectum, 38, 687-694. https://doi.org/10.1007/BF02048023

[11] Walker, A.J., Leicester, R.J., Nicholls, R.J. and Mann, C.V. (1990) A Prospective Study of Infrared Coagulation, Injection and Rubber Band Ligation in the Treatment of Haemorrhoids. International Journal of Colorectal Disease, 5, 113-116. https://doi.org/10.1007/BF00298482

[12] Shanmugam, V., Thaha, M.A., Rabindranath, K.S., Campbell, K.L., Steele, R.J.C. and Loudon, M.A. (2005) Systematic Review of Randomized Trials Comparing Rubber Band Ligation with Excisional Haemorrhoidectomy. British Journal of Surgery, 92, 1481-1487. https://doi.org/10.1007/s10350-004-6543-Z

[13] Peng, B.C., Jayne, D.G. and Ho, Y.H. (2003) Randomized Trial of Rubber Band Ligation vs. Stapled Hemorrhoidectomy for Prolapsed Piles. Diseases of the Colon \& Rectum, 46, 291-297.

[14] Alonso-Coello, P., Guyatt, G., Heels-Ansdell, D., et al. (2005) Laxatives for the Treatment of Hemorrhoids. Cochrane Database of Systematic Reviews, 19, CD004649. https://doi.org/10.1002/14651858.CD004649.pub2

[15] Alonso-Coello, P., Mills, E., Heels-Ansdell, D., et al. (2006) Fiber for the Treatment of Hemorrhoids Complications: A Systematic Review and Meta-Analysis. The American Journal of Gastroenterology, 101, 181-188. https://doi.org/10.1111/j.1572-0241.2005.00359.x

[16] Perera, N., Liolitsa, D., Iype, S., et al. (2011) Phlebotonics for Haemorrhoids. Cochrane Database of Systematic Reviews, 8, CD004322. https://doi.org/10.1002/14651858.CD004322.pub2

[17] Madoff, R.D. and Fleshman, J.W. (2004) American Gastroenterological Association Technical Review on the Diagnosis and Treatment of Hemorrhoids. Gastroenterology, 126, 1463-1473. https://doi.org/10.1053/j.gastro.2004.03.008

[18] Gupta, P.J. (2003) Infrared Coagulation versus Rubber Band Ligation in Early Stage Hemorrhoids. Brazilian Journal of Medical and Biological Research, 36, 1433-1439. https://doi.org/10.1590/S0100-879X2003001000022

[19] Jutabha, R., Jensen, D.M. and Chavalitdhamrong, D. (2009) Randomised Prospective Study of Endoscopic Rubber Bad Ligation Compared with Bipolar Coagulation for Chronical Bleeding Internal Hemorroids. American Journal of Gastroenterology, 104, 2057-2064. ttps://doi.org/10.1038/ajg.2009.292

[20] Bat, L., Melzer, E., Koler, M., Dreznick, Z. and Shemesh, E. (1993) Complications of Rubber Band Ligation of Symptomatic Internal Hemorroids. Diseases of the Colon \& Rectum, 36, 287-290. https://doi.org/10.1007/BF02053512 